\title{
Opacity in the upper atmosphere of AU Mic
}

\author{
D. S. Bloomfield ${ }^{1}$, M. Mathioudakis ${ }^{1}$, D. J. Christian ${ }^{2}$, F. P. Keenan ${ }^{1}$, and J. L. Linsky ${ }^{3}$
}

${ }^{1}$ Department of Pure and Applied Physics, The Queen's University of Belfast, Belfast, BT7 1NN, Northern Ireland, UK

${ }^{2}$ Department of Physics and Astronomy, Johns Hopkins University, 3400 North Charles Street, Baltimore, MD 21218, USA

3 JILA, University of Colorado, Boulder, CO 80309; and National Institute of Standards and Technology, USA

Received 30 January 2002 / Accepted 6 May 2002

\begin{abstract}
In this paper we investigate the validity of the optically thin assumption in the transition region of the late-type star AU Mic. We use Far-Ultraviolet Spectroscopic Explorer (FUSE) observations of the $\mathrm{C}_{\mathrm{III}}$ multiplet and O vi resonance lines, hence yielding information at two different levels within the atmosphere. Significant deviations from the optically thin fluxes are found for $\mathrm{C}_{\mathrm{III}}$ in both quiescent and flare spectra, where only $60 \%$ of the flux is actually observed. This could explain the apparent deviation of $\mathrm{C}_{\text {III }}$ observed in emission measure distributions. We utilize escape probabilities for both homogeneous and inhomogeneous geometries and calculate optical depths as high as 10 for the C III $1175.71 \AA$ component of the multiplet. Using a lower limit to the electron density $\left(10^{11} \mathrm{~cm}^{-3}\right)$ we derive an effective thickness of $<100 \mathrm{~km}$ for the scattering layer. The emission originates from very small and compact regions, consistent with a filling factor of $10^{-5}$ derived for the flare plasma.
\end{abstract}

Key words. atomic data - stars: activity - stars: atmospheres - stars: individual: AU Mic - stars: late-type - ultraviolet: stars

\section{Introduction}

In the low density and high temperature environment of transition regions and coronae, the emission is usually optically thin. Although the optical depths are sufficiently low that photons will not be trapped, a scattering layer between the emitting layer and the observer can cause photons to be scattered out of the line-of-sight thus reducing the line intensity. In such conditions strong resonance lines will appear weaker relative to forbidden transitions of the same ion. Opacity effects in the solar corona have been known for many years. Acton \& Catura (1976) provided the first convincing evidence that radiative transfer effects can be seen in X-rays. Schrijver et al. (1994) emphasized that, under certain conditions, resonant scattering can be significant in the corona of cool stars. However, an initial investigation based on Chandra observations show no appreciable coronal opacity on the RS CVn binary Capella (Phillips et al. 2001).

Based on observations obtained with the Hubble Space Telescope (HST), Mathioudakis et al. (1999) have found measurable opacity for the transition region plasma in YZ CMi becoming more significant during a flare event. The higher resolution observations of AU Mic by Robinson et al. (2001) revealed that the $10^{5} \mathrm{~K}$ slow moving plasma $\left(<60 \mathrm{~km} \mathrm{~s}^{-1}\right)$ is basically optically thin, but suggests that the faster moving plasma $\left(100 \mathrm{~km} \mathrm{~s}^{-1}\right)$ may show opacity effects.

The discrepancies in the observed line ratios of the $\mathrm{C}_{\text {III }}$ $1175 \AA$ and Si III $1206 \AA$ multiplets, observed in the solar

Send offprint requests to: $\mathrm{D}$. S. Bloomfield,

e-mail: s.bloomfield@qub.ac.uk transition region, have often been attributed to opacity (Doyle \& McWhirter 1980; Keenan \& Kingston 1986). Both studies demonstrate noticeable optical thickness near the solar limb. In late-type stars the emission measure derived from the $\mathrm{C}_{\mathrm{III}}$ $1175 \AA$ line often deviates from the average emission measure distribution, and it has been speculated that this is due to opacity (Byrne et al. 1987). However, a thorough investigation of this effect is lacking from the literature. In the present paper, we use FUSE observations to study the effects of transition region opacity on the dMe star AU Mic (HD $197481=$ GJ 803). At a distance of $10 \mathrm{pc}, \mathrm{AU}$ Mic is one of the brightest sources in its class and a prime early candidate for FUSE. We use the method of escape probabilities to evaluate optical depths, and show that these can provide information on the scattering layer of the upper stellar atmosphere.

\section{Method}

Opacity can affect both the intensities and widths of spectral lines. In the case of pure thermal broadening in a homogeneous, optically thin, medium, the optical depth at line center is given by (Mitchell \& Zemansky 1961):

$\tau_{0}=1.16 \times 10^{-14} \lambda f_{i j} \sqrt{\frac{M}{T}} \frac{n_{\mathrm{i}}}{n_{\mathrm{el}}} \frac{n_{\mathrm{el}}}{n_{\mathrm{H}}} \frac{n_{\mathrm{H}}}{n_{\mathrm{e}}} n_{\mathrm{e}} l$

where $\lambda$ is the wavelength in $\AA, f_{i j}$ the oscillator strength of the transition, $M$ the mass of the absorbing atom in atomic mass units, $T$ the temperature in $\mathrm{K}, l$ the path length in $\mathrm{cm}$ and $n_{\mathrm{i}}$, $n_{\mathrm{el}}, n_{\mathrm{H}}, n_{\mathrm{e}}$ the number densities in $\mathrm{cm}^{-3}$ of ions in the lower level $i$, element, hydrogen and free electrons, respectively. For 
Table 1. The line transitions used in the present study.

\begin{tabular}{llll}
\hline \hline Ion $(\AA)$ & Transition & $f_{i j}$ & $\begin{array}{l}A_{j i} \\
\left(10^{8} \mathrm{~s}^{-1}\right)\end{array}$ \\
\hline $\mathrm{C}_{\text {III }}(1175.71)^{a}$ & $2 \mathrm{~s} 2 \mathrm{p}^{3} \mathrm{P}_{2}-2 \mathrm{p}^{2}{ }^{3} \mathrm{P}_{2}$ & 0.20 & 9.4 \\
$\mathrm{C}_{\text {III }}(1174.94)^{b}$ & $2 \mathrm{~s} 2 \mathrm{p}^{3} \mathrm{P}_{1}-2 \mathrm{p}^{2}{ }^{3} \mathrm{P}_{2}$ & 0.11 & 3.1 \\
$\mathrm{O}_{\text {VI }}(1031.91)^{a}$ & $2 \mathrm{~s}^{2} \mathrm{~S}_{1 / 2}-2 \mathrm{p}^{2} \mathrm{P}_{3 / 2}$ & 0.131 & - \\
$\mathrm{O}_{\text {VI }}(1037.61)^{b}$ & $2 \mathrm{~s}^{2} \mathrm{~S}_{1 / 2}-2 \mathrm{p}^{2} \mathrm{P}_{1 / 2}$ & 0.065 & - \\
\hline
\end{tabular}

${ }^{a}$ Optically thick line.

${ }^{b}$ Optically thin line.

$f_{i j}$ and $A_{j i}$-values from Wiese et al. (1966).

a given plasma temperature and density, lines with large oscillator strengths will have higher optical depths, and hence suffer a greater reduction in flux, compared to fine-structure transitions with lower $f$-values. For ease, the line with the higher oscillator strength will be termed optically thick, with corresponding optical depth $\tau_{0}$, while that with the lower oscillator strength will be termed optically thin, with optical depth $\tau_{\text {thin }}$.

When the intensity ratio is used to study opacity, the lines used fall into two categories: pairs of lines arising from a common upper level and pairs sharing a common lower level. We assume collisional excitation by electrons throughout. In the first case, the observed line flux ratio will be the ratio of their $A$-values reduced by the ratio of the photon escape probabilities:

$\frac{F_{\text {thick }}}{F_{\text {thin }}}=\frac{P_{\text {thick }}}{P_{\text {thin }}} \frac{A_{\text {thick }}}{A_{\text {thin }}}$

where $F_{\text {thick }}$ and $F_{\text {thin }}$ are the line fluxes, $P_{\text {thick }}$ and $P_{\text {thin }}$ the escape probabilities and $A_{\text {thick }}$ and $A_{\text {thin }}$ the corresponding spontaneous radiative decay rates. In optically thin conditions the escape probabilities become unity.

In the second case, the line flux ratio will be equal to the ratio of their collision strengths. The relation between collision strength, $\Omega_{i j}$, and oscillator strength, $f_{i j}$, for a transition between levels $i$ and $j$ can be approximated as:

$\Omega_{i j}=\frac{8 \pi}{\sqrt{3}} \frac{I_{\mathrm{H}}}{\Delta E_{i j}} g \omega_{i} f_{i j}$

where $I_{\mathrm{H}}$ is the ionization energy for hydrogen, $g$ the Gaunt factor, $\omega_{i}$ the statistical weight of level $i$ and $\Delta E_{i j}$ the threshold energy for the transition. This formula is based on the Bethe approximation and neglects the short range interactions between the perturber and the atomic electron (van Regemorter 1962). Hence, the observed line flux ratio will be equal to the ratio of their oscillator strengths reduced by the photon escape probability. This yields:

$\frac{F_{\text {thick }}}{F_{\text {thin }}}=\frac{P_{\text {thick }}}{P_{\text {thin }}} \frac{f_{\text {thick }}}{f_{\text {thin }}}$

where $f_{\text {thick }}$ and $f_{\text {thin }}$ are the corresponding oscillator strengths (Schmelz et al. 1997).

In Table 1 we list the atomic parameters for the transitions used in this work. The $\mathrm{C}_{\text {III }}$ lines belong to the first category, while the $\mathrm{O}$ vi lines belong to the second category.
An estimate of the escape probability requires some geometry considerations. There are two possible geometries that we will consider (see Kastner \& Kastner 1990). The first requires a homogeneous distribution of emitters and absorbers. This refers to identically distributed emitters and absorbers coexisting within the same region. For a homogeneous distribution, the photon escape probability is given by:

$$
\begin{aligned}
P\left(\tau_{0}\right) & =\frac{1}{\tau_{0} \sqrt{\pi}} \int_{-\infty}^{\infty}\left\{1-\exp \left[-\tau_{0} \mathrm{e}^{-x^{2}}\right]\right\} \mathrm{d} x \\
& =\sum_{m=0}^{\infty}\left(-\tau_{0}\right)^{m}\left[(m+1) !(m+1)^{\frac{1}{2}}\right]^{-1},
\end{aligned}
$$

where $\tau_{0}$ is the optical depth at line center, $x$ the dimensionless frequency variable $x=\frac{\gamma-v_{0}}{\Delta v_{\mathrm{D}}}$ and $\Delta v_{\mathrm{D}}$ the half-width of the thermal Doppler line profile.

The second geometry is that of an inhomogeneous distribution, where the emitters and absorbers are spatially distinct. Using the same variables as in Eq. (5), the photon escape probability for an inhomogeneous distribution has the form:

$$
\begin{aligned}
P\left(\tau_{0}\right) & =\frac{1}{\tau_{0} \sqrt{\pi}} \int_{-\infty}^{\infty} \exp \left[-x^{2}-\tau_{0} \mathrm{e}^{-x^{2}}\right] \mathrm{d} x \\
& =1+\sum_{n=1}^{\infty}(-1)^{n} \tau_{0}^{n}\left[n !(n+1)^{\frac{1}{2}}\right]^{-1} .
\end{aligned}
$$

Having determined the ratio of escape probabilities from the observed line ratio, this will be used to evaluate $\tau_{0}$. In turn this will be used to obtain a column density for the scattering region, allowing evaluation of an effective thickness if the average local electron density is known.

From Eq. (1) we can see that for closely spaced lines of the same ion, relative optical depths $\left(\tau_{\text {rel }}\right)$ will depend only on $n_{\mathrm{i}} f_{i j}$. Since the $\mathrm{C}_{\text {III }}$ lines considered share a common upper level, lower level populations are required to determine $n_{\mathrm{i}}$ and hence the relation between the thick and thin line optical depths.

To calculate level populations, we have constructed a model ion for $\mathrm{C}_{\text {III }}$ consisting of the 10 energetically lowest finestructure levels, namely $2 \mathrm{~s}^{2}{ }^{1} \mathrm{~S} ; 2 \mathrm{~s} 2 \mathrm{p}{ }^{3} \mathrm{P}_{0,1,2},{ }^{1} \mathrm{P} ; 2 \mathrm{p}^{2}{ }^{3} \mathrm{P}_{0,1,2},{ }^{1} \mathrm{D}$ and ${ }^{1} \mathrm{~S}$. Energies for all these levels were taken from Moore (1993). Electron impact excitation rates for transitions among the above fine-structure levels were obtained from Berrington (1985) and Keenan et al. (1992). Einstein A-coefficients for allowed transitions were taken from Froese Fischer (1994), except for the $2 \mathrm{~s}^{2}{ }^{1} \mathrm{~S}-2 \mathrm{~s} 2 \mathrm{p}{ }^{1} \mathrm{P}$ resonance line, where the experimental value of Reistad \& Martinson (1986) was adopted. Similarly, for the $2 \mathrm{~s}^{2}{ }^{1} \mathrm{~S}-2 \mathrm{~s} 2 \mathrm{p}^{3} \mathrm{P}_{1}$ intercombination transition, the experimental $A$-value of Doerfert et al. (1997) was used. For forbidden transitions we have employed the calculations of Jönsson et al. (1998) and Fleming et al. (1996). Proton excitation rates, which will only be important for transitions within $2 s 2{ }^{3} \mathrm{P}$ (Seaton 1964), were obtained from Ryans et al. (1998).

Using the above atomic data in conjunction with the statistical equilibrium code of Dufton (1977), relative C III level populations were derived at the temperature of maximum formation, $T=6.3 \times 10^{4} \mathrm{~K}$, for an electron density of $n_{\mathrm{e}}=$ $10^{11.5} \mathrm{~cm}^{-3}$ (see Sect. 4). Details of the procedures involved and approximations made may be found in Dufton (1977) and 
Table 2. Oscillator strengths and relative optical depths for the $\mathrm{C}_{\mathrm{III}}$ $1175 \AA$ multiplet transitions.

\begin{tabular}{llll}
\hline \hline$J-J^{\prime}$ & $\lambda(\AA)$ & $f_{i j}$ & $\tau_{\text {rel }}$ \\
\hline $1-2^{1}$ & 1174.94 & 0.11 & 1.69 \\
$0-1$ & 1175.27 & 0.26 & 1.34 \\
$1-1$ & 1175.59 & 0.065 & 1.00 \\
$2-2^{2}$ & 1175.71 & 0.20 & 5.14 \\
$1-0$ & 1175.99 & 0.087 & 1.34 \\
$2-1$ & 1176.37 & 0.065 & 1.67 \\
\hline & 977.02 & 0.81 & 29.31 \\
\hline
\end{tabular}

${ }^{1}$ Optically thin line.

${ }^{2}$ Optically thick line.

Dufton et al. (1978). Values of $\tau_{\text {rel }}$ estimated from these level populations are given in Table 2 , and show ${ }^{\mathrm{C}}{ }^{\mathrm{III}} \tau_{\text {thin }} \approx \frac{\tau_{0}}{3.04}$. Since the OVI lines share a common lower level, and hence $n_{\mathrm{i}}$, the optical depth relationship is more easily found and is simply that of the $f$-values, ${ }^{\mathrm{O} V \mathrm{I}} \tau_{\text {thin }}=\frac{\tau_{0}}{2.02}$. These optical depth relations were used to evaluate numerically $\frac{P\left(\tau_{0}\right)}{P\left(\tau_{\text {thin }}\right)}$ from Eqs. (5) and (7) (Fig. 1) which were in turn used to estimate the optical depths in the homogeneous and inhomogeneous approximations.

\section{Observations and analysis}

We have examined a set of AU Mic observations obtained from the FUSE public data archive at the Multimission Archive at the Space Telescope Science Institute (MAST). The FUSE instrument consists of four separate but co-aligned primefocus telescopes. Two telescopes are optimized for the 905 $1105 \AA$ region with $\mathrm{SiC}$ coatings; the other 2 use LiF coatings to cover the 987-1187 $\AA$ region. Holographically ruled gratings disperse light onto 2 separate microchannel plate detectors (MCP's) each with 2 independent segments. Details on the FUSE instrument and in-orbit performance can be found in Moos et al. (2000) and Sahnow et al. (2000a, 2000b).

FUSE observations of AU Mic started on 26 August 2000 at 19:35 UT and data were acquired in time-tag mode. In time-tag mode photons are recorded with an $X$ and $Y$ position and pulse height. The on-board computer assigns times to events with a resolution of $1 \mathrm{~s}$. The AU Mic data were reduced with the currently available version (2.0.5) of the calfuse pipeline. Spectra were extracted from the large aperture (LWRS) and these were background subtracted, flat-fielded, wavelength and flux calibrated as described in the calfuse reference guide (van Dixon et al. 2001). The data obtained by FUSE covered 9 satellite orbits, and timing analysis shows that the first eight orbits observed AU Mic in quiescence while a flare occurred in the ninth orbit (Fig. 2). Data were therefore divided into a quiescent spectrum (orbits 1-8) and a flare spectrum (orbit 9). Exposure times were $\sim 16 \mathrm{ks}$ and $\sim 2 \mathrm{ks}$ for the quiescent state and flare, respectively. All spectra were binned to a 4-pixel resolution which corresponds to $\sim 8 \mathrm{~km} \mathrm{~s}^{-1}$, approximately $\frac{1}{2}$ of a formal resolution element. The C III $1175 \AA$
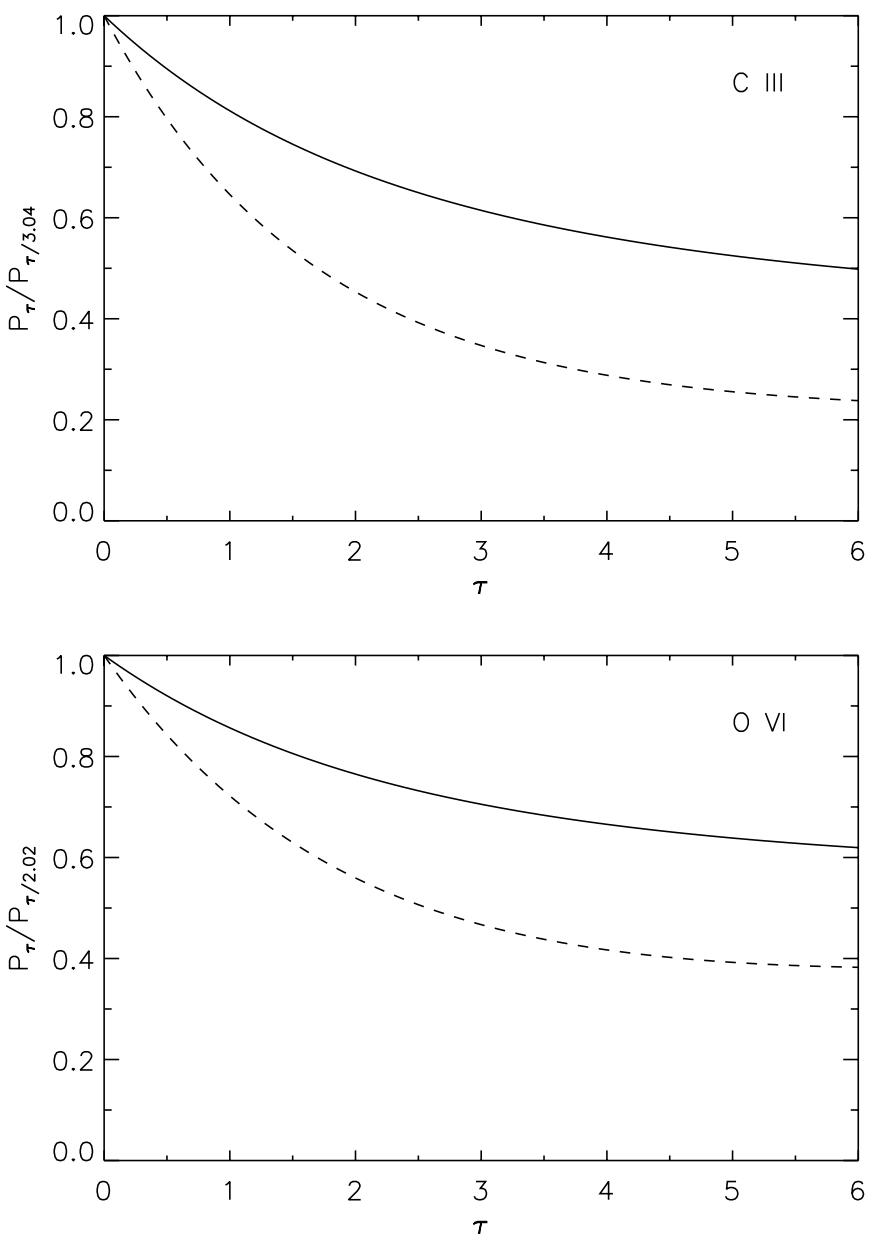

Fig. 1. Top: the ratio of escape probabilities as a function of optical depth for the $\mathrm{C}$ III case. Bottom: the ratio of escape probabilities as a function of optical depth for the O vi case. N.B. The solid curves are for homogeneous distributions of emitters and absorbers, while the dashed curves are for inhomogeneous distributions.

multiplet (Fig. 3) was observed in two spectra, while the O vI 1032 \& $1038 \AA$ resonance lines (Fig. 4) were covered by four spectra. During the flare, the C III $1175 \AA$ line flux increased by a factor of $\approx 18$.

Line fluxes were evaluated by fitting Gaussians to the observed line profiles. The multiple Gaussian fitting technique used for the 6 component $\mathrm{C}$ III multiplet involved 5 separate curves. Since the (2-2) and (1-1) transitions are blended, these were fitted by a single curve. The central wavelengths of each Gaussian were left as free parameters while the profile widths were set to be equal, with the actual value of the $F W H M$ left as a free parameter. Even though the (1-1) component will have a non-negligible contribution to the (2-2) component, if opacity effects are observed from the adopted line flux then we will be slightly underestimating the optical depth derived from the (2-2) contribution. In the case of the $\mathrm{C}_{\text {III }} 1175 \AA$ multiplet, single Gaussian profiles were sufficient to fit the individually resolved lines. However, increased red-wing emission components were observed in the $\mathrm{O}$ vi lines, where the fitting procedure required two individual components. Table 3 shows the results obtained. The $\mathrm{C}_{\text {III }} 977.02 \AA\left(2 \mathrm{~s}^{2}{ }^{1} \mathrm{~S}_{0}-2 \mathrm{~s} 2 \mathrm{p}^{1} \mathrm{P}_{1}\right)$ resonance line was observed in two spectra and this line flux was 


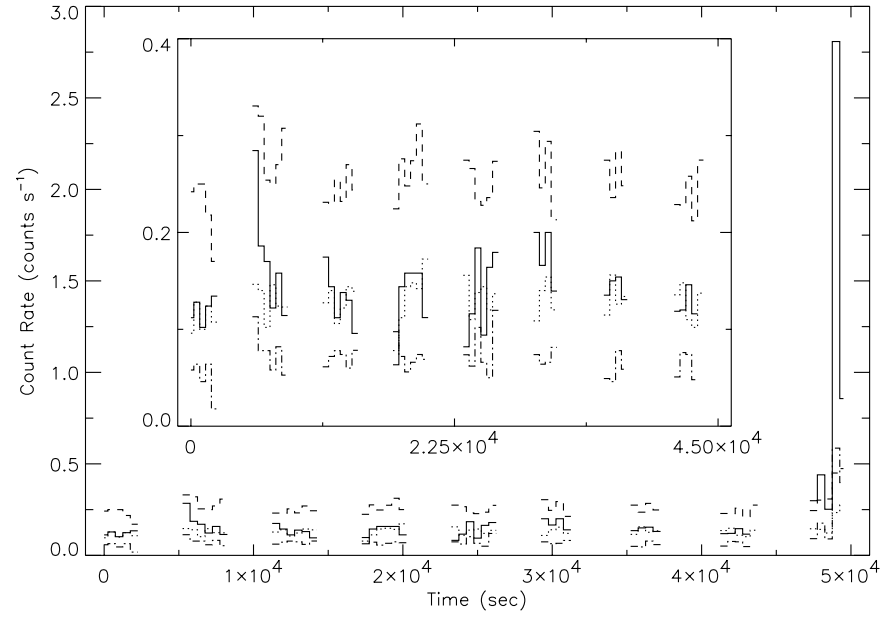

Fig. 2. Plot of complete light-curves observed on AU Mic on August 2000. Linestyles correspond to the following lines: solid $-\mathrm{C}_{\mathrm{III}}$ $1175 \AA$; dotted - O vi $1038 \AA$; dashed - O VI $1032 \AA$; dash-dot - C III $977 \AA$. Note: the inserted plot shows quiescent light-curve variability over orbits $1-8$.

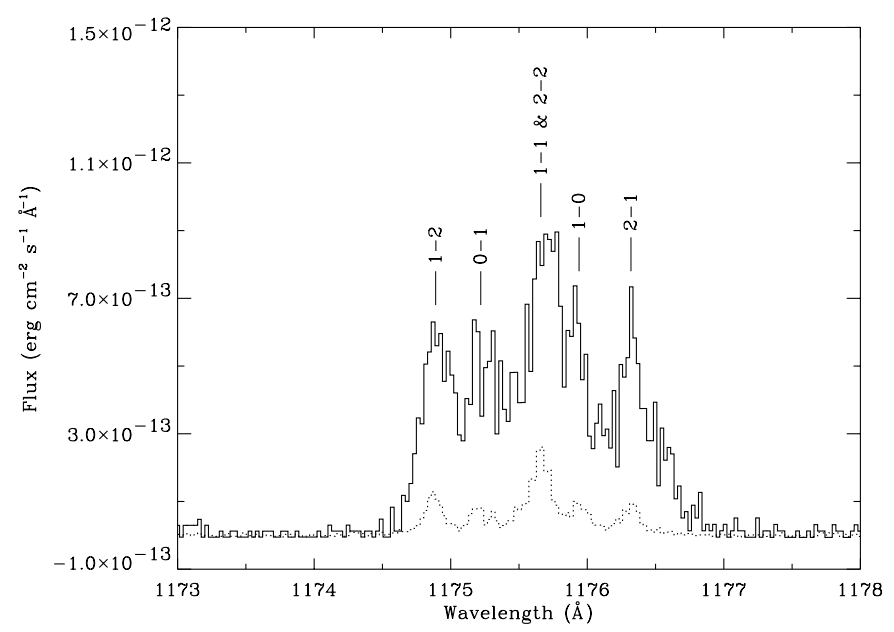

Fig. 3. C III multiplet lines observed by the $2 \mathrm{~A} \mathrm{LiF}$ detector, labeled by their $J-J^{\prime}$ values. The solid plot is the observation taken during the flare (containing both flare and quiescent components), while the dotted plot was taken during quiescence.

also evaluated as part of the fitting process, again using two Gaussian profiles due to the existence of heightened red-wing emission (see Fig. 5).

Inspection of Table 3 reveals that the $\mathrm{C}$ III line pair shows deviations from the theoretical optically thin value. The large errors involved with the $\mathrm{O} v$ I line ratios are a result of compound percentage errors arising from the fitting process. Since each line flux is determined from two Gaussian profiles which contribute two errors, and a ratio is taken introducing two further errors, the quiescent line ratio involves the addition of four percentage errors. The problem is exacerbated in the flare case with the subtraction of the quiescent component, introducing two further errors, giving four percentage errors for each line. Thus, when a ratio is taken, the total error in the $\mathrm{O}$ vi flare contribution is the addition of eight percentage errors. The $\mathrm{C}$ III line ratios show smaller errors since each line was fitted using a sin-

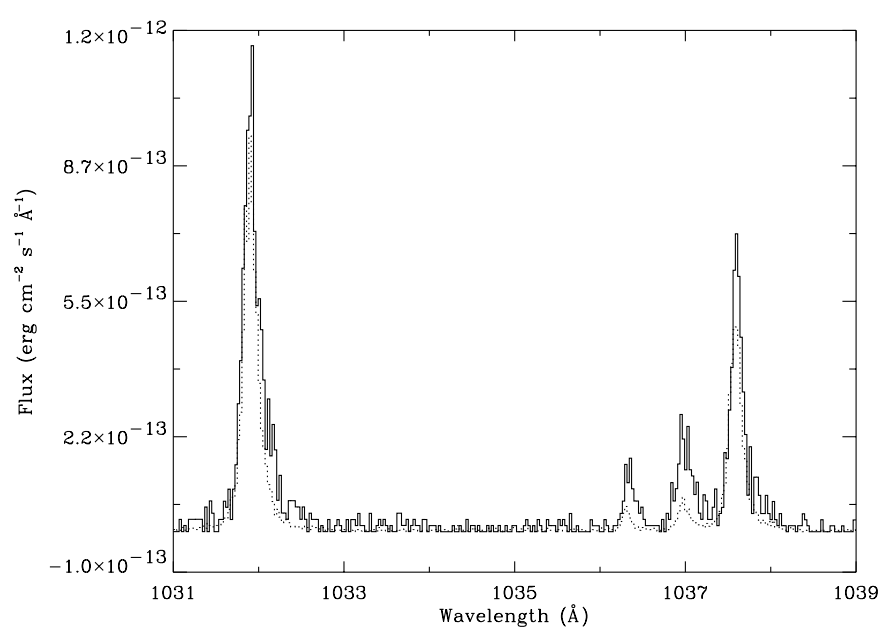

Fig. 4. $\mathrm{O}$ vi resonance lines observed by the $1 \mathrm{~A} \mathrm{LiF}$ detector. The solid plot is the observation taken during the flare (containing both flare and quiescent components), while the dotted plot was taken during quiescence.

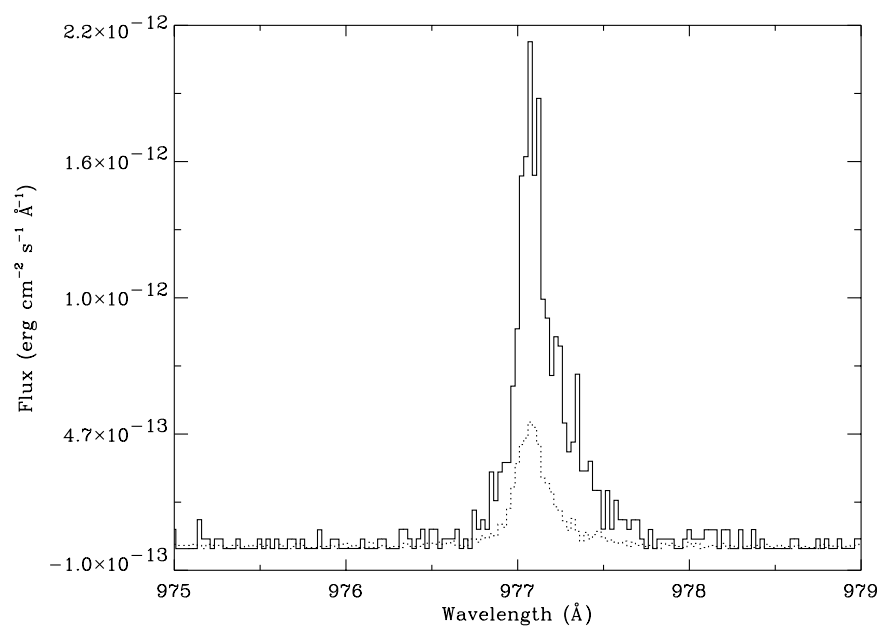

Fig. 5. $\mathrm{C}$ III resonance line observed by the $2 \mathrm{~A} \mathrm{SiC}$ detector. The solid plot is the observation taken during the flare (containing both flare and quiescent components), while the dotted plot was taken during quiescence.

gle Gaussian. Thus, the error in the quiescent line ratio involves the addition of only two percentage errors while the flare line ratio involves the addition of four percentage errors.

\section{Results and discussion}

C III $1175 \AA$ multiplet line ratios in the solar transition region are at the optically thin limit $(3: 1)$, except at heights near the limb where they reach values as low as $\approx 1.5$ (Doyle \& McWhirter 1980). In the case of the active M dwarf YZ CMi, Si IV and C IV line ratios are near their optically thin limit (2:1) in the quiescent state. However, the Si IV ratio becomes as low as 1.2 during a flare (Mathioudakis et al. 1999).

The line ratios used in this study show no $n_{\mathrm{e}}$ or $T_{\mathrm{e}}$ dependence over the $10^{6}-10^{14} \mathrm{~cm}^{-3}$ and $10^{4}-10^{7} \mathrm{~K}$ ranges (CHIANTI database; Dere et al. 1997). Observed deviations from the optically thin approximation must therefore be 
Table 3. Line fluxes and ratios of the $\mathrm{C}_{\text {III }}$ multiplet and $\mathrm{O}$ vI resonance lines observed on AU Mic. The fluxes are given in units of $10^{-14} \mathrm{erg} \mathrm{cm}^{-2} \mathrm{~s}^{-1}$ followed by the estimated error in brackets. The fluxes given for the flare contribution are the total observed fluxes (flare plus quiescent) minus the quiescent components.

\begin{tabular}{|c|c|c|c|c|c|c|c|c|c|c|c|c|}
\hline \multirow{2}{*}{$\begin{array}{l}\text { Ion } \\
(\AA)\end{array}$} & \multicolumn{2}{|c|}{$\mathrm{C}_{\text {III }}$} & \multirow[t]{2}{*}{ Ratio } & \multirow{2}{*}{$\frac{P_{\text {thick }}}{P_{\text {thin }}}$} & \multicolumn{2}{|c|}{$\tau_{1175.7}$} & \multicolumn{2}{|c|}{$\mathrm{O}_{\mathrm{VI}}$} & \multirow[t]{2}{*}{ Ratio } & \multirow{2}{*}{$\begin{array}{l}P_{\text {thick }} \\
P_{\text {thin }}\end{array}$} & \multicolumn{2}{|c|}{$\tau_{1031.9}$} \\
\hline & 1175.7 & 1174.9 & & & Hom. & Inh. & 1031.9 & 1037.6 & & & Hom. & Inh. \\
\hline Quiet & $5.0(0.1)$ & $2.3(0.1)$ & $2.18(0.11)$ & 0.72 & 1.74 & 0.74 & $19.3(3.1)$ & $9.9(2.1)$ & $1.95(0.73)$ & 0.97 & 0.21 & 0.10 \\
\hline Flare & $21.1(1.1)$ & $15.9(1.0)$ & $1.32(0.16)$ & 0.44 & 10.83 & 2.12 & $7.8(2.7)$ & $3.7(1.7)$ & $2.10(1.71)$ & 1.04 & - & - \\
\hline
\end{tabular}

due to opacity. The ratio of escape probabilities may hence be determined from Eqs. (2) and (4) and these are also given in Table 3. $\mathrm{C}$ III line ratios show large deviations from their optically thin values in quiescence, and these deviations increase during the flare. However, the O vi ratios do not deviate from their optically thin values.

At the temperatures of maximum formation in ionization equilibrium, $\log T_{\mathrm{CIII}}=4.8$ and $\log T_{\mathrm{OVI}}=5.5$, the ionic fractions are $\frac{n_{\mathrm{CII}}}{n_{\mathrm{C}}}=10^{-0.063}$ and $\frac{n_{\mathrm{OVI}}}{n_{\mathrm{O}}}=10^{-0.621}$ (Mazzotta et al. 1998). The elemental abundances are $\frac{n_{\mathrm{C}}}{n_{\mathrm{H}}}=10^{-3.40}$, $\frac{n_{\mathrm{O}}}{n_{\mathrm{H}}}=10^{-3.07}$ (Feldman 1992), where $\frac{n_{\mathrm{H}}}{n_{\mathrm{e}}}$ is taken to be 0.8 and the atomic masses are $M_{\mathrm{C}}=12.01, M_{\mathrm{O}}=16.00$. The above values, combined with the estimated optical depths, allow us to determine a column density, $n_{\mathrm{e}} l$, within the atmosphere. To derive an effective thickness for the scattering region we require an average electron density. The C III $977.02 \AA$ and $1176.37 \AA$ line ratio demonstrates density dependence over the $10^{8}-5 \times 10^{10} \mathrm{~cm}^{-3}$ range. However, the observed ratio is at the high density limit in both quiescence and flare spectra, implying that $n_{\mathrm{e}}>10^{11} \mathrm{~cm}^{-3}$. We note that the $\mathrm{C}_{\text {III }} 977 \AA$ line will be substantially affected by opacity since, as can be seen from Table 2, it has the highest relative optical depth. Also, with these lines falling in different channels, comparison of their fluxes may be affected by the target star drifting in or out of the aperture. For these reasons, this lower limit to the density was verified with the $C_{\text {III }} 1175.99 \AA\left(2 \mathrm{~s} 2 \mathrm{p}^{3} \mathrm{P}_{1}-2 \mathrm{p}^{2}{ }^{3} \mathrm{P}_{0}\right)$ and $1176.37 \AA\left(2 \mathrm{~s} 2 \mathrm{p}^{3} \mathrm{P}_{2}-2 \mathrm{p}^{2}{ }^{3} \mathrm{P}_{1}\right)$ line ratio, as both of these lines have relatively low optical depths and fall in the same channel. Our density estimate is in agreement with densities determined for AU Mic of $6.3 \times 10^{10} \mathrm{~cm}^{-3}$ during quiescence (Pagano et al. 2000 ) and $1.7 \times 10^{12} \mathrm{~cm}^{-3}$ during flare activity (Robinson et al. 2001).

Adopting a value for the average electron density, and assuming that the region of $\mathrm{O}$ vi formation has a similar density, we are able to derive upper limits to the effective thicknesses of the scattering regions. During quiescence we find dimensions of ${ }^{\text {hom }} l_{\mathrm{C} \text { III }}<20 \mathrm{~km}$ and ${ }^{\text {hom }} l_{\mathrm{OVI}}<10 \mathrm{~km}$, for the homogeneous geometry, and ${ }^{\mathrm{inh}} l_{\mathrm{C} \text { III }}<7 \mathrm{~km}$ and ${ }^{\text {inh }} l_{\mathrm{OVI}}<5 \mathrm{~km}$, for the inhomogeneous geometry. During the flare event we find dimensions of hom $l_{\text {CIII }}<100 \mathrm{~km}$ for the homogeneous case and ${ }^{\text {inh }} l_{\text {CIII }}<20 \mathrm{~km}$ for the inhomogeneous case. These small dimensions are similar to those reported for YZ CMi $(\approx 5 \mathrm{~km})$ by Mathioudakis et al. (1999), who assume an inhomogeneous geometry. They are also in accord with the requirement of a very thin transition region of high column mass, as found by Houdebine \& Doyle (1994) when reproducing observed line profiles from active stellar atmospheres through semi-empirical modeling.

We have used the net flare flux of the $C_{\text {III }} 1175 \AA$ multiplet to calculate the emission measure $\left(E M=n_{\mathrm{e}}^{2} V\right)$ for the multiplet. The $E M$ is then combined with the lower limit to the electron density to derive an upper limit of $V \leq 2 \times 10^{28} \mathrm{~cm}^{3}$ for the flare emitting volume. This would imply a filling factor of $\leq 10^{-5}$. If we assume that the emission originates from a group of semi-circular loops with a cross-sectional radius $r$ which is $\approx 20 \%$ of the loop radius $R$, then $V=0.04 \pi^{2} R^{3} N$ where $N$ is the number of loops. For $N=100$ we find that $r \leq 1500 \mathrm{~km}$, while for a single loop $r \leq 7000 \mathrm{~km}$.

The $\mathrm{C}$ III model ion was also used to determine relative line strengths for the transitions of the multiplet. Assuming we can resolve one of the $\mathrm{C}$ III multiplet lines which is least affected by opacity, i.e. the (1-1) transition, we can approximate the total flux expected for the entire $1175 \AA$ multiplet in optically thin conditions. However, inspection of Fig. 3 rules out use of this due to the heavy blending of (1-1) with the (2-2) transition. Instead, the easily resolvable (0-1) line was used to scale the total expected optically thin $\mathrm{C}$ III multiplet flux. In this way we find that we only observe $60 \%$ of the flux expected from the entire multiplet for the flare component. For the $977.02 \AA$ resonance line, where the optical depths are significantly higher, we find that only $18 \%$ of the expected flux is actually observed for the flare component.

\section{Conclusions}

In optically thin conditions the flux ratio of the observed $\mathrm{C}$ III multiplet lines is equal to the ratio of their radiative transition probabilities (3:1). We presented strong evidence for opacity effects in the transition region of the active M-type dwarf AU Mic which become more significant during a flare event. In contrast, the $\mathrm{O}$ vi lines are at the optically thin limit in both quiescence and the flare.

We use the $\mathrm{C}_{\text {III }} 977.02 / 1176.37 \AA$ line ratio to estimate a lower limit to the electron density of $>10^{11} \mathrm{~cm}^{-3}$. This lower limit is then combined with the optical depths to derive the effective thicknesses of the scattering regions. During quiescence, the effective thicknesses for the $\mathrm{O}$ VI scattering region are $<10 \mathrm{~km}$ and $<5 \mathrm{~km}$, assuming homogeneous and inhomogeneous geometries, respectively. Effective thicknesses were not able to be derived for the $\mathrm{O}$ VI scattering region during the flare, as the line ratio observed was in the optically thin limit. During the flare, the effective thickness of the $\mathrm{C}$ III scattering region was seen to increase from $<20$ to $<100 \mathrm{~km}$, for 
a homogeneous atmospheric geometry, and from $<7$ to $<20 \mathrm{~km}$, for an inhomogeneous geometry. The opacity and filling factor considerations imply that the emission originates from very small and compact emitting regions.

Finally, we wish to emphasize that although the method of escape probabilities is inherently approximate, it is still a valuable tool, due to the simple nature of the physical arguments on which it is based. Escape probabilities are in good agreement with the exact radiative transfer solutions for the case of semi-infinite media, but are substantially different in the case of finite slabs (see Hubeny 2001).

Acknowledgements. DSB would like to thank the Department for Employment and Learning for Northern Ireland for a research studentship. This work is supported by the Particle Physics and Astronomy Research Council (PPARC).

\section{References}

Acton, L., \& Catura, R. C. 1976, Phil. Trans. R. Soc. London, 281, 383

Berrington, K. A. 1985, J. Phys. B, 18, L395

Byrne, P. B., Doyle, J. G., Brown, A., Linsky, J. L., \& Rodono, M. 1987, A\&A, 180, 172

Dere, K. P., Landi, E., Mason, H. E., Monsignori-Fossi, B. C., \& Young, P. R. 1997, A\&AS, 125, 149

Doerfert, J., Träbert, E., Wolf, A., Schwalm, D., \& Uwira, O. 1997, Phys. Rev. Lett., 78, 4355

Doyle, J. G., \& McWhirter, R. W. P. 1980, MNRAS, 193, 947

Dufton, P. L. 1977, Comp. Phys. Commun., 13, 25

Dufton, P. L., Berrington, K. A., Burke, P. G., \& Kingston, A. E. 1978, A\&A, 62, 111

Feldman, U. 1992, Phys. Scr., 46, 202

Fleming, J., Bell, K. L., Hibbert, A., Vaeck, N., \& Godefroid, M. R. 1996, MNRAS, 279, 1289

Froese Fischer, C. 1994, Phys. Scr., 49, 323

Houdebine, E. R., \& Doyle, J. G. 1994, A\&A, 289, 169
Hubeny, I. 2001, in Spectroscopic Challenges of Photoionized Plasmas, ed. G. Ferland, \& D. Wolf Savin, ASP Conf. Ser., 247, 197

Jönsson, P., Froese Fischer, C., \& Träbert, E. 1998, J. Phys. B, 31, 3497

Kastner, S. O., \& Kastner, R. E. 1990, J. Quant. Spectrosc. Radiat. Transfer, 44, 275

Keenan, F. P., Feibelman, W. A., \& Berrington, K. A. 1992, ApJ, 389, 443

Keenan, F. P., \& Kingston, A. E. 1986, MNRAS, 220, 493

Mathioudakis, M., McKenny, J., Keenan, F. P., Williams, D. R., \& Phillips, K. J. H. 1999, A\&A, 351, L23

Mazzotta, P., Mazzitelli, G., Colafrancesco, S., \& Vittorio, N. 1998, A\&AS, 133, 403

Mitchell, A. C. G., \& Zemansky, M. W. 1961, in Resonance Radiation and Excited Atoms (Cambridge University Press)

Moore, C. E. 1993, in CRC Handbook of Chemistry and Physics, Edition 76 (CRC Press, Boca Raton, Florida)

Moos, H. W., Cash, W. C., Cowie, L. L., et al. 2000, ApJ, 538, L1

Pagano, I., Linsky, J. L., Carkner, L., et al. 2000, ApJ, 532, 497

Phillips, K. J. H., Mathioudakis, M., Huenemoerder, D. P., et al. 2001, MNRAS, 325, 1500

Reistad, N., \& Martinson, I. 1986, Phys. Rev. A, 34, 2632

Robinson, I., Linsky, J. L., Woodgate, B. E., et al. 2001, ApJ, 554, 368

Ryans, R. S. I., Foster-Woods, V. J., Reid, R. H. G., Keenan, F. P., \& Copeland, F. 1998, A\&A, 336, 393

Sahnow, D. J., Moos, H. W., Ake, T. B., et al. 2000a, ApJ, 538, L7

Sahnow, D. J., Moos, H. W., Ake, T. B., et al. 2000b, in UV, Optical, and IR Space Telescopes and Instruments Proc. SPIE, 4013, ed. J. B. Breckinridge, \& P. Jakobsen, 334

Seaton, M. J. 1964, MNRAS, 127, 191

Schmelz, J. T., Saba, J. L. R., Chauvin, J. C., \& Strong, K. T. 1997, ApJ, 477, 509

Schrijver, C. J., van den Oord, G. H. J., \& Mewe, R. 1994, A\&A, 289, L23

van Dixon, W., Kruk, J., \& Murphy, E. 2001, Calfuse Reference Guide, http://fuse.pha.jhu.edu/analysis/calfuse.html van Regemorter, H. 1962, ApJ, 136, 906

Wiese, W. L., Smith, M. W., \& Glennon, B. M. 1966, in Atomic Transition Probabilities, National Standard Reference Data Series 